\title{
Prevalencia de infarto de miocardio en un programa de riesgo cardiovascular de una institución prestadora de salud en Armenia-Quindío
}

\author{
Carlos A. Bedoya-Ríos ${ }^{\mathrm{a}, \mathrm{b}}$, Jessika P. Mendoza-Lozano ${ }^{\mathrm{a}, \mathrm{b}}$ y Olga Alicia Nieto Cárdenas ${ }^{\mathrm{b}, \mathrm{c}, *}$ \\ a Facultad de Ciencias de la Salud, Universidad del Quindio, Armenia, Colombia \\ b Grupo de Salud Pública, Grupo de enfermedades cardiovasculares y metabólicas GECAVYME, Facultad de Ciencias de la Salud, \\ Universidad del Quindío, Armenia, Colombia \\ c Programa de Medicina, Facultad de Ciencias de la Salud, Universidad del Quindío, Armenia, Colombia
}

Recibido el 19 de junio de 2015; aceptado el 27 de enero de 2016

Disponible en Internet el 8 de abril de 2016

\section{PALABRAS CLAVE Infarto de miocardio; Riesgo cardiovascular; Factores de riesgo cardiovascular}

\begin{abstract}
Resumen
Objetivo: Identificar la prevalencia y las características del infarto agudo de miocardio en un programa de riesgo cardiovascular de una institución prestadora de servicios en salud de primer nivel en Armenia, Quindío.

Métodos: Se realizó un estudio descriptivo de corte transversal, que tomó como población las personas inscritas al programa de riesgo cardiovascular de una institución prestadora de servicios en salud de primer nivel del municipio de Armenia-Quindío, que consultaron por infarto agudo de miocardio entre el 1 de enero y 31 de diciembre de 2014; se describieron las variables en promedio, desviación estándar e intervalos de confianza, con un análisis comparativo por sexo para los factores de riesgo.

Resultados y conclusiones: En la población del programa de riesgo cardiovascular se encontraron 293 casos con antecedente de infarto agudo de miocardio, con una prevalencia de $2,49 \%$. La edad promedio en hombres fue de 66,62 años y en mujeres 69,58 años $(p=0,02$ ). El colesterol HDL en hombres fue de $39,61 \mathrm{mg} / \mathrm{dl}$ y en mujeres de $46,58 \mathrm{mg} / \mathrm{dl}(p=0,01)$. Los niveles de colesterol LDL se encontraron elevados según lo recomendado en prevención secundaria $(110,04 \mathrm{mg} / \mathrm{dl})$. El principal factor de riesgo encontrado fue la hipertensión arterial (84\%), seguido por otros como el sedentarismo $(63,48 \%)$, el sobrepeso y la obesidad $(40,61$ y $13,98 \%$ respectivamente), el hábito de fumar $(12,97 \%)$ y el consumo de alcohol $(5,46 \%)$, que fueron mayores en los hombres.

(c) 2016 Sociedad Colombiana de Cardiología y Cirugía Cardiovascular. Publicado por Elsevier España, S.L.U. Este es un artículo Open Access bajo la licencia CC BY-NC-ND (http:// creativecommons.org/licenses/by-nc-nd/4.0/).
\end{abstract}

\footnotetext{
* Autor para correspondencia.

Correo electrónico: olgalicianieto@gmail.com (O.A. Nieto Cárdenas).
} 


\section{KEYWORDS}

Myocardial infarction; Cardiovascular risk; Cardiovascular risk factors
Prevalence of myocardial infarction in a cardiovascular risk programme conducted in an institution providing health services in Armenia - Quindío

\begin{abstract}
Motivation: To identify the prevalence and characteristics of acute myocardial infarction in a cardiovascular risk programme conducted in a first-level institution providing health services in Armenia, Quindío.

Methods: Cross-sectional descriptive study including participants in a cardiovascular risk programme of conducted in an institution providing health services in Armenia, Quindío, who consulted due to acute myocardial infarction between January $1^{\text {st }}$ and December $31^{\text {st }} 2014$. Average variables, standard deviation and confidence intervals were described with a gender-based analysis for risk factors.

Results and conclusions: 293 participants of the cardiovascular risk programme had a history of acute myocardial infarction, with a prevalence of $2.49 \%$. Average age was 66.62 for men and $69.58(p=0.02)$ for women. HDL cholesterol was $39.61 \mathrm{mg} / \mathrm{dl}$ for men and $46.58 \mathrm{mg} / \mathrm{dl}(p=0.01)$ for women. LDL cholesterol levels were high according to secondary prevention recommendations $(110.04 \mathrm{mg} / \mathrm{dl})$. The main risk factor found was arterial hypertension $(84 \%)$, followed by others such as a sedentary lifestyle (63.48\%), overweight and obesity (40.61 and $13.98 \%$ respectively), smoking (12.97\%) and alcohol consumption (5.46\%), which were more frequent amongst men.

(c) 2016 Sociedad Colombiana de Cardiología y Cirugía Cardiovascular. Published by Elsevier España, S.L.U. This is an open access article under the CC BY-NC-ND license (http:// creativecommons.org/licenses/by-nc-nd/4.0/).
\end{abstract}

\section{Introducción}

Desde el punto de vista patológico, el infarto agudo de miocardio se define como la muerte de células miocárdicas debido a isquemia prolongada; la muerte celular histológica posterior no es inmediata, tarda algún tiempo en producirse, pueden requerirse de 2 a 4 horas o más para que sea completa y observarse así por examen macroscópico o microscópico postmortem. En cuanto a la clínica, el síntoma principal es el dolor opresivo, de intensidad variable, localizado en la región retroesternal, con o sin irradiación a la zona precordial, el epigastrio, la mandíbula y/o a los brazos, especialmente a la cara cubital del brazo izquierdo, o al dorso, conocido como angina de pecho. La lesión miocárdica se detecta cuando las concentraciones sanguíneas de los biomarcadores sensibles y específicos, como troponinas cardiacas (cTn) o la fracción MB de la creatincinasa (CK-MB), aumentan. Las cTn । y T son componentes del aparato contráctil de las células miocárdicas y se expresan casi exclusivamente en el corazón. También se presentan cambios electrocardiográficos en el segmento ST, onda T, o bloqueo de rama izquierda, aparición de ondas $\mathrm{Q}$ o anormalidad en la contractilidad ${ }^{1}$.

El infarto agudo de miocardio es la causa principal de muerte en personas mayores de 55 años $^{2}$ y en Colombia se ubica en las diez primeras causas de mortalidad ${ }^{3}$. En el mundo cada 39 segundos muere una persona a causa de enfermedades cardiovasculares ${ }^{4}$. En 2012, murieron 56 millones de personas, en quienes la cardiopatía isquémica fue la primera causa, representada en un $13,2 \%^{5}$; para 2008, las enfermedades cardiovasculares y la diabetes en adultos entre 30 y 70 años de edad representaron una tasa de mortalidad de 152 por 10.000 habitantes en el mundo ${ }^{6}$.
En Colombia, entre los años 2005 y 2010, la mayor tasa de mortalidad por infarto de miocardio se encontró en el centro del país; el Quindío tuvo una tasa de mortalidad por 100 mil habitantes de 74,53, cifra que lo ubicó como el tercer departamento con mayor mortalidad ${ }^{7}$. En 2012 la American Heart Association destacó que la prevalencia de enfermedades cardiovasculares, incluyendo el infarto de miocardio, tiene un comportamiento que varía con el aumento de la edad y difiere por sexo: las mujeres tienen baja prevalencia desde los 20 hasta los 59 años en comparación con los hombres, pero posteriormente esta prevalencia es similar en ambos sexos entre los 60 años y los 79 años, para luego tornarse mayor en el sexo femenino a partir de los 80 años $^{8}$.

Por otra parte, el estudio Framingham inició su recolección de datos en 1948 e incluyó personas entre 30 a 70 años de edad. Con base en los resultados de las diferentes cohortes, fue como se identificaron los factores de riesgo que se conocen; entre otros aspectos se consideran: edad, colesterol total, colesterol HDL, presión arterial sistólica y diastólica, uso de medicación antihipertensiva, tabaquismo actual, índice de masa corporal, triglicéridos, presencia o no de diabetes y estado actual ${ }^{9}$. De igual forma, el estudio INTERHEART, con un diseño de casos y controles desarrollado en 52 países, reconoció como factores de riesgo modificables: el tabaquismo, los niveles elevados de lípidos séricos, la hipertensión arterial, la diabetes mellitus, la obesidad mórbida, el sedentarismo, el bajo consumo diario de frutas y vegetales, el consumo problemático de alcohol y el índice psicosocial $^{10}$.

El presente proyecto de investigación identificó la prevalencia de infarto agudo de miocardio en la población del programa de riesgo cardiovascular en una institución 
prestadora de servicios de salud del primer nivel de atención de Armenia, Quindío, durante el año 2014.

\section{Materiales y métodos}

El estudio fue aprobado por el Comité de Bioética de la Universidad del Quindío, se firmó consentimiento informado institucional y se guardó la confidencialidad de los participantes.

\section{Población y muestra}

Se realizó un estudio descriptivo de corte transversal, tomando como población las personas que se encontraban inscritas al programa de Riesgo Cardiovascular de una institución prestadora de servicios en salud de primer nivel del municipio de Armenia-Quindío, que consultaron durante el 1 de enero al 31 de diciembre de 2014, cifra que ascendió a 11.765 .

\section{Criterios de inclusión}

1. Haber consultado durante el año 2014 al programa de riesgo cardiovascular de una institución prestadora de servicios en salud de primer nivel del municipio de Armenia-Quindío.

2. Haber presentado antecedente de infarto agudo de miocardio, lo cual se encontraba registrado en la historia clínica respaldado con reportes de electrocardiograma, ecocardiograma y/o diagnóstico consignados por el médico.

\section{Tamaño de la muestra}

Se conformó con todas las personas que tenían registrado un antecedente de infarto de miocardio en 2014 y que cumplieron con los criterios de inclusión ya descritos. En total se incluyeron 293 sujetos.

\section{Procesamiento de la información}

Se diseñó una base de datos en Excel en la que se tabuló la información correspondiente a las variables de estudio consideradas como factores de riesgo para presentar infarto agudo de miocardio: edad, sexo, peso, índice de masa corporal, perímetro abdominal, niveles de triglicéridos, colesterol total, colesterol HDL, colesterol LDL, glicemia preprandial y postprandial, tensión arterial, nivel educativo y estado civil; se incluyeron, además, variables como tabaquismo, consumo de alcohol, sedentarismo, antecedentes de hipertensión arterial y diabetes mellitus.

El procesamiento de la información se llevó a cabo en el programa Statgraphics Centurion versión 17.1.02; se describieron las variables con promedio, desviación estándar e intervalos de confianza (IC 95\%). La prevalencia de infarto de miocardio se describió en porcentaje. La información obtenida se presentó en tablas y se describieron sobre el total y por sexo, comparativamente.

El informe correspondiente a los resultados encontrados se expuso ante el Programa de Medicina de la Universidad del Quindío y al Asesor de riesgo cardiovascular de la institución prestadora de servicios en salud de primer nivel del municipio de Armenia-Quindío.

\section{Resultados}

Consultaron al programa de riesgo cardiovascular de una institución prestadora de servicios en salud de Armenia, 11.765 personas durante el año 2014, de las cuales, 664 presentaban enfermedad coronaria y 293 tuvieron antecedente de infarto agudo de miocardio, confirmado por electrocardiograma, ecocardiograma y/o registro en la historia clínica por parte del médico; la prevalencia de infarto de miocardio encontrada para ese año en el Programa de riesgo cardiovascular de una institución prestadora de servicios en salud en Armenia, fue de 2,49\%.

En la tabla 1 se observan los años de presentación de infarto de miocardio, que van desde 1994 hasta 2014; el mayor porcentaje se registró durante los años 2013 y 2014, con un $27,98 \%$.

En las características de los sujetos de investigación, la edad promedio fue de 67,87 años, el peso promedio de $65,52 \mathrm{~kg}$ y la talla promedio $156,66 \mathrm{~cm}$.

El índice de masa corporal promedio fue de $26,57 \mathrm{~kg} / \mathrm{m}^{2}$, 117 personas $(39,93 \%)$ tenían peso normal, 119 (40,61\%) sobrepeso y $41(13,98 \%)$ obesidad; 13 personas tenían bajo peso $(4,43 \%)$ (tabla 2$)$.

Tabla 1 Año de presentación del infarto agudo de miocardio

\begin{tabular}{lcc}
\hline Año & Número & Porcentaje \\
\hline 1994 & 1 & $0,34 \%$ \\
1999 & 1 & $0,34 \%$ \\
2004 & 3 & $1,02 \%$ \\
2005 & 3 & $1,02 \%$ \\
2006 & 6 & $2,05 \%$ \\
2007 & 7 & $2,39 \%$ \\
2008 & 4 & $1,37 \%$ \\
2009 & 7 & $2,39 \%$ \\
2010 & 5 & $1,71 \%$ \\
2011 & 7 & $2,39 \%$ \\
2012 & 23 & $7,85 \%$ \\
2013 & 48 & $16,38 \%$ \\
2014 & 34 & $11,60 \%$ \\
No registrado & 144 & $49,15 \%$ \\
TOTAL & 293 & $100 \%$ \\
\hline
\end{tabular}

Tabla 2 Índice de masa corporal

\begin{tabular}{lcc}
\hline Índice de masa corporal & Número & Porcentaje \\
\hline Bajo peso & 13 & $4,43 \%$ \\
Peso normal & 117 & $39,93 \%$ \\
Sobrepeso & 119 & $40,61 \%$ \\
Obesidad tipo 1 & 31 & $10,58 \%$ \\
Obesidad tipo 2 & 6 & $2,04 \%$ \\
Obesidad mórbida & 4 & $1,36 \%$ \\
No registrado & 3 & $1,02 \%$ \\
Total & 293 & $100 \%$ \\
\hline
\end{tabular}


Tabla 3 Resultados de las variables cuantitativas estudiadas

\begin{tabular}{lcc}
\hline Variable & $\begin{array}{c}\text { Promedio } \pm \text { desviación } \\
\text { estándar }\end{array}$ & IC 95\% \\
\hline Edad (años) & $67,87 \pm 10,78$ & $66,63-69,11$ \\
Peso (kg) & $65,62 \pm 19,75$ & $63,34-67,90$ \\
Talla (cm) & $156,66 \pm 17,05$ & $154,69-158,63$ \\
$\begin{array}{c}\text { Índice de masa } \\
\text { corporal }\end{array}$ & $26,57 \pm 9,61$ & $25,46-27,68$ \\
$\begin{array}{c}\text { Perímetro } \\
\text { abdominal (cm) }\end{array}$ & $92,95 \pm 14,12$ & $91,76-94,77$ \\
$\begin{array}{c}\text { Tensión arterial } \\
\text { diastólica } \\
\text { (mm Hg) }\end{array}$ & $76,60 \pm 11,96$ & $75,23-77,98$ \\
$\begin{array}{c}\text { Tensión arterial } \\
\text { sistólica (mm Hg) }\end{array}$ & $126,71 \pm 24,57$ & $123,88-129,53$ \\
$\begin{array}{c}\text { Glicemia } \\
\text { preprandial } \\
\text { (mg/dl) }\end{array}$ & $108,01 \pm 44,77$ & $101,31-114,70$ \\
$\begin{array}{c}\text { Glicemia } \\
\text { postprandial } \\
\text { (mg/dl) }\end{array}$ & $149 \pm 64,29$ & $118,01-179,98$ \\
$\begin{array}{c}\text { Colesterol total } \\
\text { (mg/d) }\end{array}$ & $185,10 \pm 42,52$ & $178,78-191,34$ \\
$\begin{array}{c}\text { Triglicéridos } \\
\text { (mg/dL) }\end{array}$ & $188,09 \pm 110,56$ & $171,04-205,13$ \\
$\begin{array}{c}\text { Colesterol LDL } \\
\text { (mg/dl) }\end{array}$ & $110,04 \pm 39,63$ & $103,92-116,15$ \\
$\begin{array}{c}\text { Colesterol HDL } \\
\text { (mg/dl) }\end{array}$ & $42,37 \pm 15,11$ & $40,04-44,70$ \\
\hline & & \\
\hline
\end{tabular}

El perímetro abdominal promedio fue de $92,95 \mathrm{~cm}$; las cifras tensionales promedio correspondieron a $126,71 / 76,6 \mathrm{~mm} \mathrm{Hg}$, es decir, estaban en niveles normales.

El perfil lipídico promedio fue el siguiente: colesterol total en $185,10 \mathrm{mg} / \mathrm{dL}$; triglicéridos en $188,09 \mathrm{mg} / \mathrm{dL}$, colesterol HDL 42,37 mg/dL y colesterol LDL $110,04 \mathrm{mg} / \mathrm{dL}$. Los niveles de glicemia, pre- y postprandial, se encontraron en 108,01 y $101,31 \mathrm{mg} / \mathrm{dL}$ respectivamente (tabla 3 ).

En cuanto a los factores de riesgo presentes, el $12,97 \%$ eran fumadores, el $63,48 \%$ sedentarios, el 5,46\% consumidores de alcohol, mientras que el $84,36 \%$ y el $21,84 \%$ tenía antecedente personal de hipertensión arterial y diabetes mellitus, respectivamente (tabla 4).

De acuerdo con el nivel educativo, el 60,07\% (176) cursó hasta básica primaria, el $19,8 \%$ no cursó ningún nivel educativo, $12,29 \%$ cursó básica secundaria, $2,73 \%$ cursó sólo hasta preescolar, 1,02\% aprobó la media académica clásica, 1,02\% tenía un nivel profesional de educación, 0,34\% poseía un nivel académico técnico y en el $2,39 \%$ no se registró nivel educativo.

En lo que respecta a distribución de acuerdo con el estado civil, $32,08 \%$ eran casados, $22,53 \%$ viudos, $19,45 \%$ solteros y $15,02 \%$ vive en unión libre.

El 57,62\% (169) correspondió al sexo masculino y el $42,32 \%$ (124) al femenino (tabla 5).

Las variables estudiadas que presentaron una diferencia estadísticamente significativa $(p<0,05)$ por sexo fueron las siguientes: edad promedio en hombres de 66,62 $\pm 11,19$ años $y$ en mujeres $69,58 \pm 9,99$ años $(p=0,02)$; tensión arterial sistólica promedio en hombres de $124,06 \pm 22,57 \mathrm{~mm} \mathrm{Hg}$ y en mujeres de $130,31 \pm 26,73 \mathrm{~mm} \mathrm{Hg}(p=0,03)$; colesterol total promedio en hombres de $177,29 \pm 40,99 \mathrm{mg} / \mathrm{dL}$ y en mujeres de $196,4 \pm 42,41 \mathrm{mg} / \mathrm{dL}(\mathrm{p}=0,01)$; y colesterol HDL promedio en hombres de $39,61 \pm 11,06 \mathrm{mg} / \mathrm{dL}$ y en mujeres de $46,58 \pm 19,10 \mathrm{mg} / \mathrm{dL}(p=0,01)$. No se registró diferencia significativa en el índice de masa corporal por sexo (tabla 6).

Respecto a los factores de riesgo, el tabaquismo fue de $12,97 \%$, más frecuente en hombres $(9,56 \%)$ que en mujeres $(3,41 \%)$; el sedentarismo representó el $63,48 \%$, siendo las mujeres $(32,76 \%)$ más sedentarias que los hombres $(30,73 \%)$; en cuanto al consumo de alcohol, fue de $5,46 \%$, siendo los hombres $(4,78 \%)$ más consumidores que las mujeres $(0,68 \%)$ (tabla 7).

\section{Discusión}

\section{Prevalencia}

En la población de estudio se halló una prevalencia de 2,49\% de infarto agudo de miocardio. A la fecha no se tiene información previa acerca de la prevalencia del infarto agudo de miocardio en años anteriores en esta población ni del municipio, que permita comparar la cardiopatía isquémica en Armenia.

Al cotejar la prevalencia de infarto de miocardio con otra población de riesgo cardiovascular, en el estudio realizado por Rodríguez ${ }^{11}$ en el Hospital San Ignacio, se halló una prevalencia menor de infarto de miocardio $(1,33 \%)$ en contraste con la de $2,49 \%$ encontrada en este estudio. Al comparar los factores de riesgo estudiados, se reportaron cifras menores en factores de riesgo como hipertensión arterial (en este estudio 84,3\%, Rodríguez 65\%); sedentarismo (en este estudio $63,48 \%$, Rodríguez 14\%) y diabetes mellitus (en este estudio $21,84 \%$, Rodríguez 19\%). Se encontraron cifras mayores en variables como tabaquismo (en este estudio $12,97 \%$, Rodríguez $51 \%$ ) y alcoholismo (en este estudio $5,46 \%$, Rodríguez 24\%).

Si bien este estudio se llevó a cabo en una población de riesgo cardiovascular, se compara la prevalencia con estudios de población general en algunas áreas de Colombia. Por ejemplo, en el estudio Caribe, realizado entre julio y diciembre de 2003 en la Costa Caribe colombiana ${ }^{12}$, la prevalencia de infarto agudo de miocardio de $1,5 \%$, fue menor en comparación con la encontrada en nuestro estudio.

Frente al estudio CARMELA, realizado en 7 países de Latinoamérica, incluyendo Colombia ${ }^{13}$, dicha prevalencia correspondió al $2 \%$, pero cabe resaltar que incluye antecedente de ataque cerebrovascular.

A diferencia de los estudios anteriores, en el de Valdés et al., hecho en consultorios médicos en la Habana, Cuba, en $1995^{14}$, la prevalencia correspondió al 4,7\%, superior a la encontrada en el presente estudio.

\section{Características demográficas}

La edad promedio según el sexo tuvo una diferencia estadísticamente significativa $(p=0,02)$; en mujeres fue de $69,58 \pm 9,99$ años y en hombres de $66,62 \pm 11,19$ años, a pesar de encontrarse ambos en el grupo etáreo en el cual la 
Tabla 4 Factores de riesgo

\begin{tabular}{llcc}
\hline Variable & Categoría & Frecuencia & Porcentaje \\
\hline Tabaquismo & Sí & 38 & $12.97 \%$ \\
& No & 255 & $87,03 \%$ \\
Ejercicio físico & Sí & 107 & $36,52 \%$ \\
& No & 186 & $63,48 \%$ \\
Consumo alcohol & Sí & 16 & $5,46 \%$ \\
& No & 277 & $94.54 \%$ \\
Antecedente personal de & Sí & 247 & $84,3 \%$ \\
hipertensión arterial & No & 46 & $15,7 \%$ \\
Antecedente personal de & Sí & 64 & $21,84 \%$ \\
diabetes & No & 229 & $71,16 \%$ \\
\hline
\end{tabular}

Tabla 5 Distribución de la prevalencia de infarto agudo de miocardio por sexo

\begin{tabular}{lll}
\hline Sexo & Número & Porcentaje \\
\hline Masculino & 169 & $57,68 \%$ \\
Femenino & 124 & $42,32 \%$ \\
Total & 293 & $100 \%$ \\
\hline
\end{tabular}

evidencia considera que se ha nivelado el riesgo en la presentación del infarto agudo de miocardio ${ }^{8}$, es decir ambos sexos entre los 60 años y los 79 años de edad. La edad ha sido un factor determinante, considerándose que en las mujeres suele ser tardía puesto que los niveles de estrógenos son un factor protector frente al infarto agudo de miocardio ${ }^{8}$; sin embargo, después de la menopausia el riesgo de sufrir una cardiopatía isquémica es igual al de los hombres ${ }^{15}$.

\section{Factores de riesgo}

Pese a que la población participante se encuentra en control de riesgo cardiovascular, tiene hábitos más saludables y conservadores en comparación con la del estudio Caribe ${ }^{12}$, que se llevó a cabo en población general. En cuanto al consumo de alcohol se halló un $5,46 \%$ en este estudio y $15,6 \%$ en el Caribe, mientras el consumo de cigarrillo fue de $12,97 \%$ en el presente estudio y de $15,42 \%$ en el Caribe. No obstante, la población de estudio resultó ser más sedentaria $(63,48 \%)$ respecto a la del Caribe $(37,37 \%)$. El diagnóstico de hipertensión arterial fue mayor (84,3\% en este estudio y $20,96 \%$ en el (aribe), al igual que el diagnóstico de diabetes mellitus $(21,84 \%$ en este estudio, mientras que en el Caribe fue de $7,17 \%)$; sin embargo, es necesario tener en cuenta que el estudio se realizó en una población de un programa de riesgo cardiovascular.

\section{Tabaquismo}

El consumo de cigarrillo puede aumentar hasta 2,8 veces el riesgo de sufrir un infarto agudo de miocardio ${ }^{16}$; en este estudio se encontró una cifra menor $(12,97 \%)$ comparada con el Caribe $(15,42 \%)^{12}$, y menor a la documentada por González et al. $(21,1 \%)^{17}$.

Tabla 6 Distribución de las variables cuantitativas por sexo

\begin{tabular}{|c|c|c|c|}
\hline \multirow[t]{2}{*}{ Variable } & \multicolumn{2}{|c|}{ Promedio \pm desviación estándar } & \multirow[t]{2}{*}{ Valor de $\mathrm{p}$} \\
\hline & Masculino & Femenino & \\
\hline Peso & $67,80 \pm 11,43$ & $62,66 \pm 27,03$ & 0,0281 \\
\hline Talla & $161,33 \pm 20,55$ & $150,31 \pm 6,62$ & 0,0000 \\
\hline Índice de masa corporal & $25,71 \pm 6,73$ & $27,73 \pm 12,44$ & 0,0763 \\
\hline Perímetro abdominal & $94,20 \pm 14,20$ & $91,99 \pm 11,31$ & 0,1527 \\
\hline Tensión arterial diastólica & $75,68 \pm 9,42$ & $77,87 \pm 14,68$ & 0,1211 \\
\hline Tensión arterial sistólica & $124,06 \pm 22,57$ & $130,31 \pm 26,73$ & 0,0312 \\
\hline Glicemia prepandial & $106,08 \pm 38,53$ & $110,67 \pm 52,36$ & 0,5064 \\
\hline Glicemia posprandial & $131,75 \pm 52,51$ & $178,57 \pm 75,69$ & 0,1290 \\
\hline Colesterol total & $177,29 \pm 40,99$ & $196,4 \pm 42,41$ & 0,0031 \\
\hline Triglicéridos & $182,70 \pm 106,09$ & $195,89 \pm 117,10$ & 0,4544 \\
\hline Colesterol LDL & $105,34 \pm 39,22$ & $116,83 \pm 39,51$ & 0,0678 \\
\hline Colesterol HDL & $39,61 \pm 11,06$ & $46,58 \pm 19,10$ & 0,0036 \\
\hline
\end{tabular}


Tabla 7 Distribución de los factores de riesgo por sexo

\begin{tabular}{|c|c|c|c|c|}
\hline Variable & Categoría & Sexo & Frecuencia & Porcentaje \\
\hline \multirow[t]{2}{*}{ Sexo } & & Masculino & 169 & $57,68 \%$ \\
\hline & & Femenino & 124 & $42,32 \%$ \\
\hline \multirow{4}{*}{ Tabaquismo } & Sí & Masculino & 28 & $9,56 \%$ \\
\hline & & Femenino & 10 & $3,41 \%$ \\
\hline & No & Masculino & 141 & $48,12 \%$ \\
\hline & & Femenino & 114 & $38,91 \%$ \\
\hline & Sí & Masculino & 79 & $26,96 \%$ \\
\hline \multirow{3}{*}{ físico } & & Femenino & 28 & $9,56 \%$ \\
\hline & No & Masculino & 90 & $30,73 \%$ \\
\hline & & Femenino & 96 & $32,76 \%$ \\
\hline \multirow[t]{4}{*}{ Consumo de alcohol } & Sí & Masculino & 14 & $4,78 \%$ \\
\hline & & Femenino & 2 & $0,68 \%$ \\
\hline & No & Masculino & 155 & $52,90 \%$ \\
\hline & & Femenino & 122 & $41,64 \%$ \\
\hline \multirow{4}{*}{$\begin{array}{l}\text { Antecedente personal de } \\
\text { hipertensión arterial }\end{array}$} & Sí & Masculino & 137 & $46,76 \%$ \\
\hline & & Femenino & 110 & $37,54 \%$ \\
\hline & No & Masculino & 32 & $10,92 \%$ \\
\hline & & Femenino & 14 & $4,78 \%$ \\
\hline \multirow{4}{*}{$\begin{array}{l}\text { Antecedente personal de } \\
\text { diabetes }\end{array}$} & Sí & Masculino & 32 & $10,92 \%$ \\
\hline & & Femenino & 32 & $10,93 \%$ \\
\hline & No & Masculino & 137 & $46,76 \%$ \\
\hline & & Femenino & 92 & $31,40 \%$ \\
\hline
\end{tabular}

\section{Consumo de alcohol}

El consumo de bebidas alcohólicas $(5,46 \%)$ representó un mayor porcentaje en comparación con el estudio de González $(2,2 \%)^{17}$, pero menor que en el Caribe $(15,6 \%)^{12}$.

\section{Cifras tensionales}

El antecedente personal de hipertensión arterial en este estudio representa el $84,3 \%$ en toda la población. En el estudio de Bedoya et al. hecho en Colombia en un Hospital de Caldas ${ }^{18}$, el porcentaje de pacientes con antecedente de hipertensión arterial fue de $52,2 \%, 29,3 \%$ en hombres y $22,9 \%$ en mujeres, constituyendo un riesgo para la presencia de infarto agudo de miocardio y mortalidad por esta causa.

El antecedente de hipertensión arterial identificado en este estudio $(84,3 \%)$ es mayor al registrado en los estudios de González $(26,2 \%)^{17}$, Caribe $(20,96 \%)^{12}$ y CARMELA $(18 \%)^{13}$. Sin embargo, cabe resaltar, como ya se ha hecho, que este estudio se llevó a cabo en población de un programa de riesgo cardiovascular.

\section{Colesterol total}

Este ítem indicó valores promedio de $185,10 \pm 42,52 \mathrm{mg} / \mathrm{dl}$; en la distribución por sexo se encontró una diferencia significativa: en hombres fue de $177,29 \pm 40,99 \mathrm{mg} / \mathrm{dl}$ y en mujeres fue de $196,4 \pm 42,41 \mathrm{mg} / \mathrm{dL}(p=0,003)$. En el estudio de Rodríguez, hecho en Cuba en $1998^{19}$, se encontró un valor de colesterol mayor de $240 \mathrm{mg} / \mathrm{dl}$ en el $76,9 \%$ de los pacientes, en los hombres correspondió a un porcentaje de $48,81 \%$ y en las mujeres al $27,38 \%$. No obstante, nuestra población hace parte de un programa de riesgo cardiovascular, en el cual muchos participantes reciben hipolipemiantes.

\section{Colesterol HDL}

Las HDL, consideradas como un factor protector de riesgo cardiovascular, por ser indicador del transporte reverso de colesterol $^{20}$, estuvieron en niveles promedio de $42,37 \pm 15,11 \mathrm{mg} / \mathrm{dl}$, pero al comparar por sexo, fue evidente que en hombres se encontraron por debajo de los límites normales $(39,61 \pm 11,06 \mathrm{mg} / \mathrm{dl})$, no así en las mujeres $(46,58 \pm 19,10 \mathrm{mg} / \mathrm{dl})$, con una diferencia estadísticamente significativa $(p=0,003)$; esto corrobora estudios anteriores llevados a cabo en Armenia y en el Quindío, en los cuales se han encontrado niveles bajos de $\mathrm{HDL}^{21}$ en comparación con los recomendados de cifras mayores a $40 \mathrm{mg} / \mathrm{dL}$, dato relevante para el ATP IV ${ }^{22}$ a fin de evitar la aterosclerosis y con ello, episodios isquémicos como el infarto agudo de miocardio.

\section{Conclusiones}

Este estudio sobre prevalencia de infarto agudo de miocardio, realizado en la población del programa de riesgo cardiovascular de una institución prestadora de servicios en salud de primer nivel en el municipio de Armenia-Quindío durante 2014, determinó que consultaron 11.765 personas y se presentaron 293 casos con antecedente de infarto de miocardio, lo que representa una prevalencia del 2,49\%.

Entre las características generales de los sujetos de investigación, los hombres correspondieron al mayor porcentaje de personas que ha sufrido un evento coronario $(57,62 \%)$, pero a una edad menor que las mujeres. 
La presión arterial se halló en los parámetros adecuados, los valores de colesterol HDL estuvieron por debajo de lo normal en hombres, en tanto que se registraron valores de LDL por encima del valor recomendado en prevención secundaria.

Se evidenciaron, así mismo, como principales factores de riesgo el antecedente personal de hipertensión arterial (84\%) y el sedentarismo (63\%), el sobrepeso y la obesidad ( $40,6 \%$ y $13,98 \%$ respectivamente) y el perímetro abdominal por encima del límite recomendado. Fueron más sedentarias las mujeres $(32,76 \%)$ que los hombres $(30,73 \%)$, y estos últimos más fumadores y más consumidores de alcohol $(9,56 \%$ y $4,78 \%)$ que las primeras $(3,41 \%$ y $0,68 \%)$.

\section{Responsabilidades éticas}

Protección de personas y animales. Los autores declaran que para esta investigación no se han realizado experimentos en seres humanos ni en animales.

Confidencialidad de los datos. Los autores declaran que en este artículo no aparecen datos de pacientes.

Derecho a la privacidad y consentimiento informado. Los autores declaran que en este artículo no aparecen datos de pacientes.e los datos. Los autores declaran que en este artículo no aparecen datos de pacientes.

\section{Financiación}

Vicerrectoría de investigaciones de la Universidad del Quindío.

\section{Conflicto de intereses}

Los autores declaran no tener ningún conflicto de intereses.

\section{Agradecimientos}

A la Red Salud Armenia-ESE; al Dr. Jorge Luis Duque, Asesor del Programa de Riesgo cardiovascular; a la Dra. Diana Marcela Curtidor por su orientación en los aspectos bioéticos, y a la Vicerrectoría de Investigaciones de la Universidad del Quindío, por su apoyo para este proyecto Semillero.

\section{Bibliografía}

1. Thygesen K, Alpert J, Jaffe A, Simoons M, Chaitman B, Mendis $S$, et al. Third universal definition of myocardial infarction. Eur Heart J. 2012;33(20):2551-67.

2. Senior JM, Lugo LH, Acosta N, Acosta JL, Díaz J, Osío OH, et al. Guía de práctica clínica para el síndrome coronario agudo. Bogotá Ministerio de salud y protección social. 2012.

3. Principales causas de mortalidad en Colombia. Bogotá, Colombia: Ministerio de salud y protección social; 2010.

4. Rosamond W, Flegal K, Furie K, Go A, Greenlund K, Haase $N$, et al. Heart disease and stroke statistics-2008 update: a report from the American Heart Association Statistics Committee and Stroke Statistics Subcommittee. Circulation. 2008;117(4):25-146.
5. Organización Mundial de la Salud. Las 10 causas principales de defunción en el mundo. Ginebra: centro de prensa OMS; 2014.

6. Organización Mundial de la Salud. Estadísticas sanitarias mundiales. Ginebra: OMS; 2014.

7. Ministerio de salud y protección social. Hombres, más propensos en Colombia a morir de infarto. Bogotá: Minsalud; 2014.

8. Roger V, Go AS, Lloyd-Jones D, Benjamin EJ, Borden WB, Bravata DM, et al. Heart Disease and Stroke Statistics-2012 Update: A Report From the American Heart. Circulation. 2012;125:220.

9. D’Agostino RB, Pencina MJ, Vasan RS, Wolf PA, Kannelm WB, Massaro JM, et al. General cardiovascular risk profile for use in primary care: the Framingham Heart Study. Circulation. 2008;117(6):743-53.

10. Yusuf S, Hawken S, Ounpuu S, Dans T, Avezum A, Lanas F, et al., INTERHEART Study Investigators. Effect of potentially modifiable risk factors associated with myocardial infarction in 52 countries (the INTERHEART study): case control study. Lancet. 2004;364:937-52.

11. Rodríguez I. Factores de riesgo y prevalencia de infarto agudo de miocardio en el Hospital Universitario San Ignacio, 1999-2005. Investigación en Enfermería: Imagen y Desarrollo. 2006;8(1):88-97.

12. Manzur F, Arrieta CO. Estudio sociológico y del conocimiento de los factores de riesgo de las enfermedades cardiovasculares en la Costa Caribe Colombiana (Estudio Caribe). Rev Colomb Cardiol. 2005;12(3):122-8.

13. Schargrodsky H, Hernández R, Champagne BM, Silva H, Vinueza R, Silva Ayçaguer LC, et al. CARMELA Study Investigators. CARMELA: assessment of cardiovascular risk in seven Latin American cities. Am J Med. 2008;121:58-65.

14. Valdés EP, Morrees AA, Alonso NL. Prevalencia y factores de riesgo de cardiopatía isquémica. Rev Cubana Med Gen Integr. 1998;14(6):590-4.

15. Villasmil ER, Guerra VM, Torres MM, Reyna N, Mejía MJ. Perfil lipídico en mujeres premenopáusicas y posmenopáusicas. Rev Obstet Ginecol Venez. 2007;67(2):107-14.

16. Álvarez JT, Hernández V, Hechavarría GP, Antomarchi OD, Bolívar ME. Factores de riesgo coronarios asociados al infarto agudo de miocardio en el adulto mayor. MEDISAN. 2013;17(1): 54-60.

17. González MA, Dennis RJ, Devia JH, Echeverri D, Briceño GD, Gil F, et al. Factores de riesgo cardiovascular y de enfermedades cr,ónicas en población caficultora. Rev Salud Pública. 2012;14(3):390-403.

18. Bedoya T, Díez D, Loaiza J, Valencia J, Vergel M, Castaño 0 , et al. Estudio descriptivo sobre infarto agudo de miocardio en el Hospital de Caldas, 1996-2002. Colomb Med. 2004;35(3):127-31.

19. Rodríguez L, Herrera VG, Dorta EM. Algunos factores de riesgo que favorecen la aparición del infarto agudo de miocardio. Rev Cubana Med Gen Integr. 1998;14(3):243-9.

20. Pérez Méndez O, Lipoproteínas de alta densidad (HDL). ¿Un objetivo terapéutico en la prevención de la aterosclerosis? Arch Cardiol Méx. 2004;74(1):53-67.

21. Landazury P, Loango N, Gallego ML, Restrepo B. Diferencias de sexo, edad y lípidos plasmáticos asociadas al polimorfismo de la apolipoproteína E en un grupo de escolares de Quindío, Colom$\mathrm{bia} / G e n d e r$, age and plasma lipids differences associated with apolipoprotein E polymorphism in school children. Biomédica. 2009;29:382-91.

22. Stone N, Robinson J, Lichtenstein A, Bairey Merz C, LloydJones D, Wilson P, et al. 2013 ACC/AHA Guideline on the Treatment of Blood Cholesterol to Reduce Atherosclerotic Cardiovascular Risk in Adults. A Report of the American College of Cardiology/American Heart Association Task Force on Practice Guidelines. J Am Coll Cardiol. 2013;63:2889-934. 